\title{
Multifaceted functions of Siva-1: more than an Indian God of Destruction
}

\author{
Yide Mei $^{\boxplus}$, Mian $\mathbf{W u}$ \\ Hefei National Laboratory for Physical Sciences at Microscale and School of Life Sciences, University of Science and \\ Technology of China, Hefei 230027, China \\ $\triangle$ Correspondence: meiyide@ustc.edu.cn (Y. Mei), wumian@ustc.edu.cn (M. Wu) \\ Received January 9, 2012 Accepted February 4, 2012
}

\begin{abstract}
Siva-1, as a p53-inducible gene, has been shown to induce extensive apoptosis in a number of different cell lines. Recent evidence suggests that Siva-1 functions as a part of the auto-regulatory feedback loop that restrains p53 through facilitating Mdm2-mediated p53 degradation. Also, Siva-1 plays an important role in suppressing tumor metastasis. Here we review the current understanding of Siva-1-mediated apoptotic signaling pathway. We also add comments on the p53-Siva-1 feedback loop, the novel function of Siva-1 in suppressing tumor metastasis, and their potential implications.
\end{abstract}

KEYWORDS Siva-1, p53, apoptosis, tumor metastasis, stathmin

\section{INTRODUCTION}

Apoptosis is a cell suicide program that plays a central role in the normal development and maintenance of homeostasis. Dysregulation of apoptosis has been implicated in various human diseases, including cancer, auto-immune diseases, and neurodegenerative disorders (Thompson, 1995; Vaux and Korsmeyer, 1999). Apoptosis mainly occurs through two pathways: the death receptor (extrinsic) pathway and the mitochondrial (intrinsic) pathway (Elmore, 2007). In the death receptor pathway, upon engagement with corresponding ligands outside a cell, death receptors of the tumor necrosis factor (TNF) receptor superfamily are activated, promoting a cascade of events that eventually lead to the cell demise. The mitochondrial pathway, on the other hand, is evoked by the "intracellular" cues such as DNA damage, oncogene activa- tion, and nutrient deprivation. These intrinsic apoptotic signals converge on the mitochondria, causing mitochondrial release of death inducing factors that carry out the cell death cascade.

Siva-1 (named after the Hindu God of Destruction Shiva) was originally cloned and identified as a protein that associates with the cytoplasmic tail of CD27, a member of the TNF receptor superfamily (Fig. 1) (Prasad et al., 1997). As indicated by its name, Siva-1 has the ability to induce extensive apoptosis through both extrinsic and intrinsic apoptotic pathways in a number of different cell lines. Siva-1 is a relative small protein with a unique amphipathic helical region (SAH) at its $\mathrm{N}$ terminus, a death domain homology region (DDHR) in the central part, and a C-terminal cysteine-rich B-box- or Zinc finger-like structure (Prasad et al., 1997). An alternative splicing variant of Siva-1, Siva-2, which lacks most of the DDHR, is less apoptotic (Prasad et al., 1997; Yoon et al., 1999; Xue et al., 2002), which supports an idea that DDHR is essential for Siva-1's pro-apoptotic function. However, a recent study shows that overexpression of Siva-2 similarly induces apoptosis in T lymphocytes (Py et al., 2004). These suggest the functional complexity of Siva-1 in apoptosis regulation. In addition to binding to CD27, Siva-1 also interacts with other cell surface membrane receptors such as GITR (glucocorticoid-induced tumor necrosis factor receptor) and CD4 and mediates their apoptotic signaling upon respective ligand engagement (Py et al., 2007; Spinicelli et al., 2002). Siva-1 is also shown to associate with lysophosphatidic acid receptor-2 (LPA2 receptor) (Lin et al., 2007; E et al., 2009). This association promotes ubiquitination and subsequent degradation of Siva-1 upon LPA treatment. In addition to ubiquitination, Siva-1 is subjected to the phosphorylation by c-Abl-related tyrosine kinase ARG, thereafter promoting ARG-mediated apoptosis during oxidative stress (Cao et al., 2001). Moreover, tyrosine kinase 2 is reported to phosphorylate Siva-1 and 


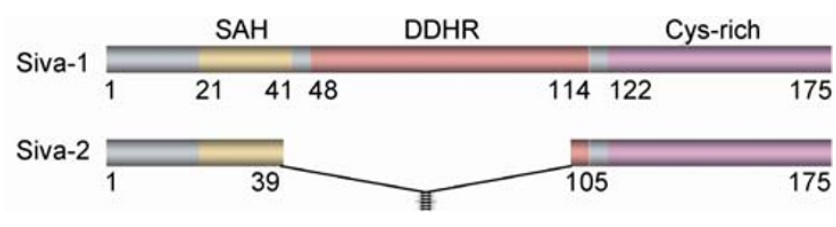

Figure 1. The schematic representation of Siva-1 and Siva-2.

enhance its apoptotic properties (Shimoda et al., 2010).

Several studies have shown that Siva-1 is overexpressed in various pathological situations, such as acute ischemic injury and coxsachievirus infection (Padanilam et al., 1998; Henke et al., 2000, 2001). Moreover, Siva-1 gene expression is also upregulated in response to DNA damage signals and hypoxia conditions (Daoud et al., 2003; Walmsley et al., 2011). Ectopic expression of a metastasis suppressor Tip30/CC3 activates Siva-1 transcription in small cell lung carcinoma (Xiao et al., 2000). The transcription factors, including p53 and E2F1, are reported to directly bind to the promoter of Siva-1 and transcriptionally activate Siva-1 expression (Fortin et al., 2004). As a result, Siva-1 enhances DNA damage-induced apoptosis (Chu et al., 2005; Barkinge et al., 2009). Also, Siva-1 is essential for p53-dependent apoptosis in cerebella granule neurons (Jacobs et al., 2007). In addition to inducing apoptosis of $\mathrm{T}$ cell lines through a caspase-dependent mitochondrial apoptotic pathway (Py et al., 2004), Siva-1 may also be involved in inhibition of NFKB activation during $T$ cell receptor-mediated activation-induced cell death (Gudi et al., 2006; Resch et al., 2009). Siva-1 is shown to interact with $\mathrm{Bcl}-2 / \mathrm{Bcl}-\mathrm{xL}$ and abrogate their anti-apoptotic activity, therefore sensitizing cells to UV radiation-induced apoptosis (Xue et al., 2002). Furthermore, the SAH region of Siva- 1 alone appears to be both necessary and sufficient to interact with $\mathrm{Bcl}-2 / \mathrm{Bcl}-\mathrm{xL}$ and enhance UVor cisplatin-induced apoptosis (Xue et al., 2002; Chu et al., 2005).

\section{AN AUTO-REGULATORY FEEDBACK LOOP OF p53-Siva-1}

As above-mentioned, Siva-1 is induced by p53. The tumor suppressor p53 maintains the normal cell growth and genomic stability by either imposing a cell cycle arrest or inducing apoptosis in response to DNA damage or other types of cell stress (Vogelstein et al., 2000; Vousden and Lu, 2002). In unstressed cells, the levels of p53 are strictly restrained by its major E3 ligase Mdm2 (Haupt et al., 1997; Kubbutat et al., 1997). Following DNA damage, ATM (ataxia-telangiectasia mutated)-mediated phosphorylation leads to destabilization of Mdm2, therefore triggering p53 stabilization and activation (Maya et al., 2001). Once activated, p53 induces expression of a large number of genes including Siva-1 and Mdm2 that regulates apoptosis and cell cycle progression (Beckerman and Prives, 2010).
Intriguingly, our recent data reveals an important role of Siva-1 as an adaptor for Mdm2 to inhibit p53 (Du et al., 2009). In unstressed cells, Siva-1 promotes the p53-Mdm2 interaction and enhances Mdm2-mediated p53 ubiquitination and degradation without affecting the stability of Mdm2. Although Siva-1 is well documented for its pro-apoptotic function, we have shown the compelling evidences that Siva-1 exerts a potent inhibitory effect on p53 activity. When expressed ectopically, Siva-1 strongly inhibits p53-mediated gene expression, apoptosis, and cell growth inhibition, whereas downregulation of Siva-1 shows the opposite effect. In xenograft mouse tumor models, Siva-1 knockdown leads to a dramatic decrease in tumor formation correlating with p53 upregulation in those tumors (Du et al., 2009). These suggest, as a p53-inducible gene, Siva-1 may be part of the auto-regulatory feedback loop that inhibits p53 activity after a non-lethal stress response. Interestingly, RAP80 (receptor associated protein 80 ), another p53-responsive gene, is also shown to negatively regulate p53 activity via promoting Mdm2-mediated p53 degradation, reinforcing the importance of the auto-inhibitory regulation of p53 (Yan et al., 2009). The discrepancy between our recent findings and previous reports may reflect the intrinsic ability of Siva-1 to regulate multiple signaling pathways and indicate the complexity of Siva-1 function. Therefore, how Siva- 1 functions in regulating apoptosis, particularly when cells are exposed to the different levels of cellular stress, e.g. non-lethal or lethal stress signals, deserves further careful investigation. Nonetheless, taken all the evidence together, we present a model for the role of Siva-1 in regulating p53 stability shown in Fig. 2. It is not unconceivable that in response to non-lethal cellular stress, Siva- 1 is upregulated by p53, which in turn functions together with Mdm2 and inhibits p53 activity, establishing a fail-safe mechanism to keep cells alive. However, the lethal stress may trigger the signal strong enough to induce apoptosis. In this case, the p53-Siva-1-Mdm2 complex is disrupted and Siva-1 may lose its ability to inhibit p53 via Mdm2.

\section{A NEW ROLE OF Siva-1 IN TUMOR METASTASIS}

Tumor metastasis is depicted as a process in which cancer cells spread from a primary tumor of origin to colonize distant organs (Chambers et al., 2002). Epithelial-mesenchymal transition (EMT), a process by which epithelial cells undergo a phenotypic conversion that gives rise to mesenchymal cells, is now believed to play a pivotal role in regulating tumor metastasis (Polyak and Weinberg, 2009; Thiery et al., 2009). We have recently revealed an important function of Siva-1 in the regulation of EMT and metastasis of tumor cells (Fig. 3) (Li et al., 2011). Upregulation of Siva-1 suppresses, while knockdown of Siva-1 enhances, both EMT and tumor metastasis. Siva-1 is shown to interact with stathmin, a microtubule-destabilizing phosphoprotein. Stathmin depoly- 

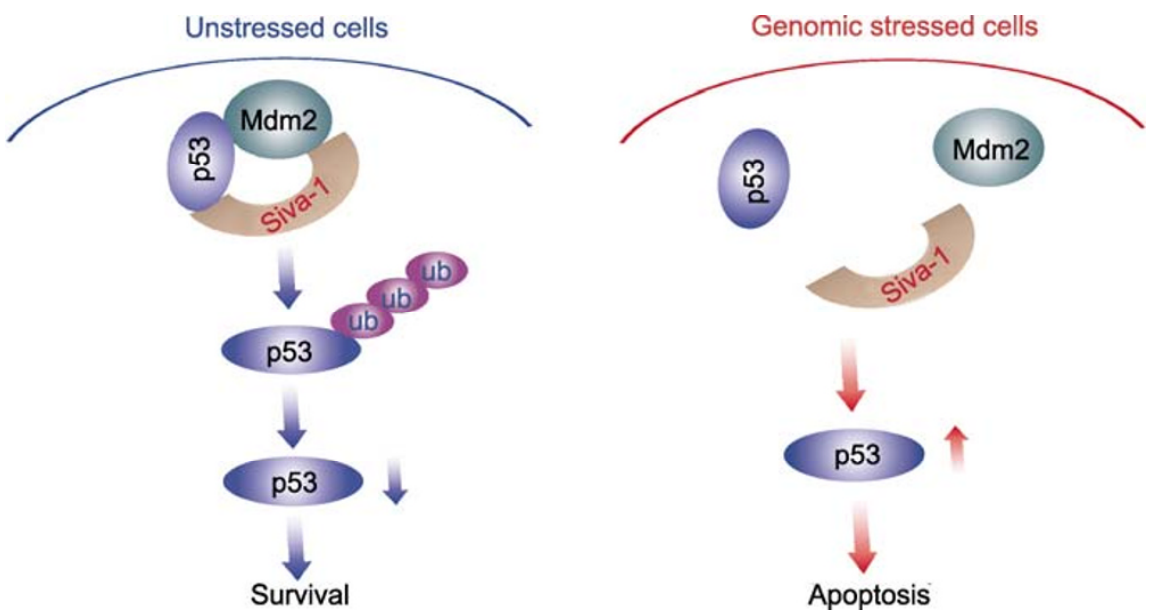

Figure 2. A model for the role of Siva-1 in regulating p53 stability. In unstressed cells, Siva-1 promotes the p53-Mdm2 interaction and enhances Mdm2-mediated p53 ubiquitination and degradation. However, genomic stress signals promote the disruption of Mdm2-Siva-1-p53 ternary complex, thus leading to the stabilization of p53.

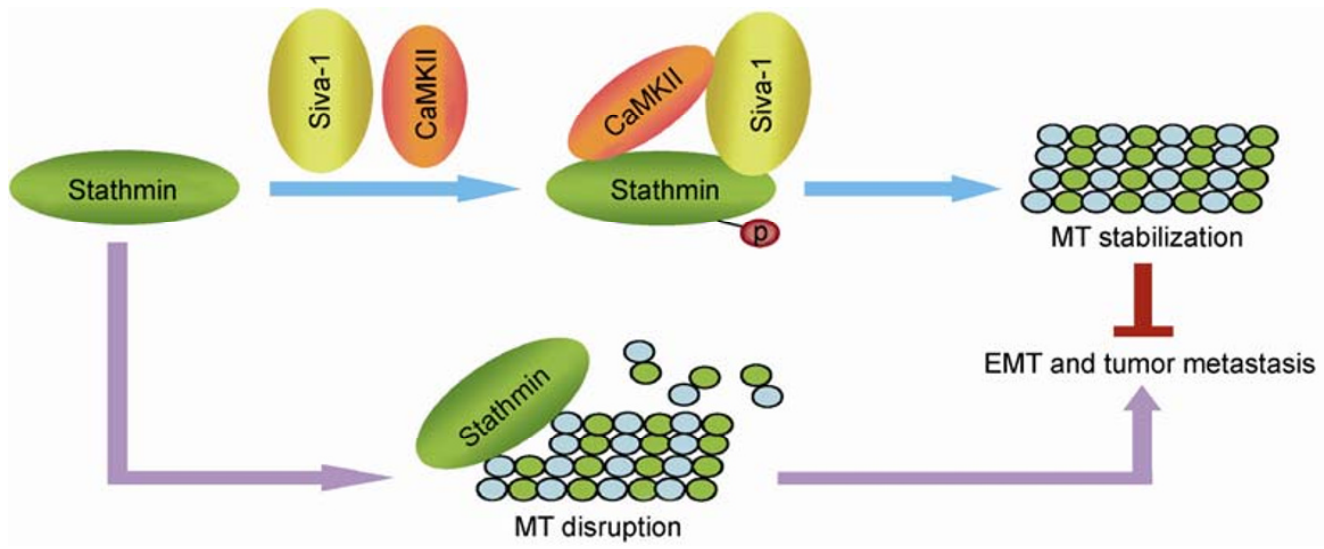

Figure 3. A model for the role of Siva-1 in suppressing EMT and tumor metastasis. Via forming a ternary complex with stathmin and CaMKII, Siva-1 inhibits the microtubules-destabilizing activity of stathmin, directly through binding, as well as indirectly through promoting phosphorylation of stathmin by CaMKII. This leads to the stabilization of microtubule and the suppression of EMT and tumor metastasis. EMT, epithelial mesenchymal transition.

merizes microtubules by either sequestering free $\alpha / \beta$-tubulin heterodimers or directly inducing microtubule catastrophe. It has been shown that phosphorylation of its $\mathrm{N}$-terminal region, particularly at Ser16 and Ser63, strongly decreases the ability of stathmin to bind to and sequester soluble tubulin and its ability to act as a catastrophe factor by directly binding to the microtubules (Manna et al., 2009). Therefore, the activity of stathmin appears to be regulated primarily at two levels: its association with the tubulin dimmers and its phosphorylation status. Siva-1 is shown to inhibit the microtubules-destabilizing activity of stathmin, directly through binding, as well as indirectly through promoting phosphorylation of stathmin at Ser16 by $\mathrm{Ca}^{2+} /$ Calmodulin-dependent protein kinase II (CaMKII). Via inhibition of stathmin, Siva-1 stabilizes microtubule, and suppresses focal adhesion assembly, cell migra- tion, and EMT (Li et al., 2011). These observations suggest that Siva- 1 is a novel suppressor in regulating EMT and tumor metastasis. In accordance with this, low levels of Siva-1 are linked to the progression and metastasis of human breast tumors (Li et al., 2011). Since current anti-cancer drug discovery and development has been highly relying on the identification of key players in controlling tumor metastasis and understanding of their involved pathways, these findings also raise an intriguing possibility that Siva-1 may be a promising therapeutic target for novel anti-tumor and anti-metastatic therapies.

Stathmin has been shown to be highly expressed in a variety of human cancers, including acute leukemia, lymphoma, Wilm's tumor, and non-small cell lung cancers (Rana et al., 2008). Although no noticeable difference in stathmin level 
was found between metastatic and non-cancerous breast tumor, metastatic breast tumors, however, indeed express much lower levels of both Siva-1 and pS16-stathmin than normal or low metastatic breast tumors. This suggests that Siva-1 and pS16-stathmin may be biomarkers and therapeutic targets for breast cancer (Li et al., 2011). Therefore it would be particularly interesting to know whether Siva-1 and pS16-stathmin are also downregulated in the other human cancers, by knowing that, Siva-1 and pS16-stathmin may represent a more general phenomenon in cancers.

\section{CONCLUDING REMARKS}

Siva-1, since identified as a CD27-binding protein, has been almost exclusively studied in the context of apoptosis regulation. When expressed ectopically, Siva-1 is capable of inducing extensive apoptosis through multiple mechanisms dependent on the cellular context. Intriguingly, as a p53-inducible gene, Siva-1 also functions as a part of the autoregulatory feedback loop that suppresses p53 by promoting Mdm2-mediated p53 degradation. A recent study also demonstrates a previously unexpected role of Siva- 1 in negatively regulating tumor metastasis through inhibition of stathmin activity. These findings indicate that the function of Siva-1 is much more complex than previously thought, which would prompt a more in-depth investigation of Siva-1 function and its involved signaling pathways. Given the crucial role of Siva-1 in the regulation of apoptosis and tumor metastasis, Siva-1 may represent as a potential valuable target for therapeutic intervention of cancer.

\section{FUTURE PERSPECTIVES}

The human Siva gene is located to chromosome 14 (q32-33, negative strand) (Xue et al., 2002). Interestingly, this region is often targeted for chromosomal translocation $t$ (14:18) (q32:q21) seen in different types of human lymphoid malignancies (Korsmeyer, 1999). The potential role of Siva-1 in these cancers remains to be determined. As an important regulator of apoptosis and metastasis, Siva-1 has been found to be downregulated in colorectal cancer and breast cancers (Okuno et al., 2001; Li et al., 2011), indicating a tumor suppressor function of Siva-1. However, whether the levels of Siva-1 are also lower in other types of cancer remains unanswered.

The Siva gene generates the predominant full-length Siva-1 and the minor alternative splice form Siva-2. Siva-2 lacks the exon 2 coding for SAH region of Siva-1, because of which Siva-2 appears to be less apoptotic. It would be interesting to know whether Siva-2 behaves as a dominant negative inhibitor of Siva-1. More importantly, whether the levels of Siva-2 are dysregulated in human cancers and whether the ratio of Siva-1/Siva-2 acts as one of the determinants for tumor development requires further examination.
As mentioned earlier, Siva-1 was initially identified as a CD27-interacting protein and was considered to be a cytosolic protein. Subsequent studies indicate that the cytosolic localization of Siva-1 seems to be essential for its function in regulating apoptosis and tumor metastasis (Spinicelli et al., 2002; Xue et al., 2002; Jacobs et al., 2007). However, our findings, as well as previous reports, support an idea that Siva-1 localizes mainly in nucleus (Py et al., 2004; Du et al., 2009; Li et al., 2011). Therefore, further exploration of nuclear Siva-1 function would be of great importance to advance our understanding of basic biology of Siva-1.

Although Siva-1 has been clearly implicated in the regulation of tumor metastasis, the underlying molecular mechanism still needs to be further investigated. Increasing evidence suggests that wide-type p53 opposes EMT and cell migration to prevent metastasis. However, mutant p53 appears to acquire additional functions to drive cell migration, invasion, and metastasis (Muller et al., 2011). Therefore the p53-Siva-1 feedback loop makes it particularly interesting to investigate whether Siva-1 mediates the inhibitory effect of p53 on tumor metastasis and whether the status of p53 affects the function of Siva-1 in regulating tumor metastasis.

\section{ACKNOWLEDGEMENTS}

This work was supported by the National Natural Science Foundation of China (Grant Nos. 31030046 and 30871290) to MW and the Ministry of Science and Technology of China (No. 2010CB912804).

\section{ABBREVIATIONS}

ATM, ataxia-telangiectasia mutated; CaMKII, Calmodulin-dependent protein kinase II; DDHR, death domain homology region; EMT, epithelial-mesenchymal transition; GITR, glucocorticoid-induced tumor necrosis factor receptor; NFkB, nuclear factor kappa B; TNF, tumor necrosis factor

\section{REFERENCES}

Barkinge, J.L., Gudi, R., Sarah, H., Chu, F., Borthakur, A., Prabhakar, B.S., and Prasad, K.V. (2009). The p53-induced Siva-1 plays a significant role in cisplatin-mediated apoptosis. J Carcinog 8, 2.

Beckerman, R., and Prives, C. (2010). Transcriptional regulation by p53. Cold Spring Harb Perspect Biol 2, a000935.

Cao, C., Ren, X., Kharbanda, S., Koleske, A., Prasad, K.V., and Kufe, D. (2001). The ARG tyrosine kinase interacts with Siva-1 in the apoptotic response to oxidative stress. J Biol Chem 276, 11465-11468.

Chambers, A.F., Groom, A.C., and MacDonald, I.C. (2002). Dissemination and growth of cancer cells in metastatic sites. Nat Rev Cancer 2, 563-572.

Chu, F., Barkinge, J., Hawkins, S., Gudi, R., Salgia, R., and Kanteti, P.V. (2005). Expression of Siva-1 protein or its putative amphipathic helical region enhances cisplatin-induced apoptosis in breast cancer cells: effect of elevated levels of BCL-2. Cancer Res 65, 
5301-5309.

Daoud, S.S., Munson, P.J., Reinhold, W., Young, L., Prabhu, V.V., Yu, Q., LaRose, J., Kohn, K.W., Weinstein, J.N., and Pommier, Y. (2003). Impact of p53 knockout and topotecan treatment on gene expression profiles in human colon carcinoma cells: a pharmacogenomic study. Cancer Res 63, 2782-2793.

Du, W., Jiang, P., Li, N., Mei, Y., Wang, X., Wen, L., Yang, X., and $\mathrm{Wu}$, M. (2009). Suppression of p53 activity by Siva1. Cell Death Differ 16, 1493-1504.

E, S., Lai, Y.J., Tsukahara, R., Chen, C.S., Fujiwara, Y., Yue, J., Yu, J.H., Guo, H., Kihara, A., Tigyi, G., et al. (2009). Lysophosphatidic acid 2 receptor-mediated supramolecular complex formation regulates its antiapoptotic effect. J Biol Chem 284, 14558-14571.

Elmore, S. (2007). Apoptosis: a review of programmed cell death. Toxicol Pathol 35, 495-516.

Fortin, A., MacLaurin, J.G., Arbour, N., Cregan, S.P., Kushwaha, N., Callaghan, S.M., Park, D.S., Albert, P.R., and Slack, R.S. (2004). The proapoptotic gene SIVA is a direct transcriptional target for the tumor suppressors p53 and E2F1. J Biol Chem 279, 28706-28714.

Gudi, R., Barkinge, J., Hawkins, S., Chu, F., Manicassamy, S., Sun, Z., Duke-Cohan, J.S., and Prasad, K.V. (2006). Siva-1 negatively regulates NF-kappaB activity: effect on T-cell receptor-mediated activation-induced cell death (AICD). Oncogene 25, 3458-3462.

Haupt, Y., Maya, R., Kazaz, A., and Oren, M. (1997). Mdm2 promotes the rapid degradation of p53. Nature 387, 296-299.

Henke, A., Launhardt, H., Klement, K., Stelzner, A., Zell, R., and Munder, T. (2000). Apoptosis in coxsackievirus B3-caused diseases: interaction between the capsid protein VP2 and the proapoptotic protein siva. J Virol 74, 4284-4290.

Henke, A., Nestler, M., Strunze, S., Saluz, H.P., Hortschansky, P., Menzel, B., Martin, U., Zell, R., Stelzner, A., and Munder, T. (2001). The apoptotic capability of coxsackievirus B3 is influenced by the efficient interaction between the capsid protein VP2 and the proapoptotic host protein Siva. Virology 289, 15-22.

Jacobs, S.B., Basak, S., Murray, J.I., Pathak, N., and Attardi, L.D. (2007). Siva is an apoptosis-selective p53 target gene important for neuronal cell death. Cell Death Differ 14, 1374-1385.

Korsmeyer, S.J. (1999). BCL-2 gene family and the regulation of programmed cell death. Cancer Res 59, 1693s-1700s.

Kubbutat, M.H., Jones, S.N., and Vousden, K.H. (1997). Regulation of p53 stability by Mdm2. Nature 387, 299-303.

Li, N., Jiang, P., Du, W., Wu, Z., Li, C., Qiao, M., Yang, X., and Wu, M. (2011). Siva1 suppresses epithelial-mesenchymal transition and metastasis of tumor cells by inhibiting stathmin and stabilizing microtubules. Proc Natl Acad Sci U S A 108, 12851-12856.

Lin, F.T., Lai, Y.J., Makarova, N., Tigyi, G., and Lin, W.C. (2007). The lysophosphatidic acid 2 receptor mediates down-regulation of Siva-1 to promote cell survival. J Biol Chem 282, 37759-37769.

Manna, T., Thrower, D.A., Honnappa, S., Steinmetz, M.O., and Wilson, L. (2009). Regulation of microtubule dynamic instability in vitro by differentially phosphorylated stathmin. J Biol Chem 284, 15640-15649.

Maya, R., Balass, M., Kim, S.T., Shkedy, D., Leal, J.F., Shifman, O.,
Moas, M., Buschmann, T., Ronai, Z., Shiloh, Y., et al. (2001). ATM-dependent phosphorylation of Mdm2 on serine 395: role in p53 activation by DNA damage. Genes Dev 15, 1067-1077.

Muller, P.A., Vousden, K.H., and Norman, J.C. (2011). p53 and its mutants in tumor cell migration and invasion. J Cell Biol 192, 209-218.

Okuno, K., Yasutomi, M., Nishimura, N., Arakawa, T., Shiomi, M., Hida, J., Ueda, K., and Minami, K. (2001). Gene expression analysis in colorectal cancer using practical DNA array filter. Dis Colon Rectum 44, 295-299.

Padanilam, B.J., Lewington, A.J., and Hammerman, M.R. (1998). Expression of CD27 and ischemia/reperfusion-induced expression of its ligand Siva in rat kidneys. Kidney Int 54, 1967-1975.

Polyak, K., and Weinberg, R.A. (2009). Transitions between epithelial and mesenchymal states: acquisition of malignant and stem cell traits. Nat Rev Cancer 9, 265-273.

Prasad, K.V., Ao, Z., Yoon, Y., Wu, M.X., Rizk, M., Jacquot, S., and Schlossman, S.F. (1997). CD27, a member of the tumor necrosis factor receptor family, induces apoptosis and binds to Siva, a proapoptotic protein. Proc Natl Acad Sci U S A 94, 6346-6351.

Py, B., Bouchet, J., Jacquot, G., Sol-Foulon, N., Basmaciogullari, S., Schwartz, O., Biard-Piechaczyk, M., and Benichou, S. (2007). The Siva protein is a novel intracellular ligand of the CD4 receptor that promotes HIV-1 envelope-induced apoptosis in T-lymphoid cells. Apoptosis 12, 1879-1892.

Py, B., Slomianny, C., Auberger, P., Petit, P.X., and Benichou, S. (2004). Siva-1 and an alternative splice form lacking the death domain, Siva-2, similarly induce apoptosis in T lymphocytes via a caspase-dependent mitochondrial pathway. J Immunol 172, $4008-4017$.

Rana, S., Maples, P.B., Senzer, N., and Nemunaitis, J. (2008). Stathmin 1: a novel therapeutic target for anticancer activity. Expert Rev Anticancer Ther 8, 1461-1470.

Resch, U., Schichl, Y.M., Winsauer, G., Gudi, R., Prasad, K., and de Martin, R. (2009). Siva1 is a XIAP-interacting protein that balances NFkappaB and JNK signalling to promote apoptosis. J Cell Sci 122, 2651-2661.

Shimoda, H.K., Shide, K., Kameda, T., Matsunaga, T., and Shimoda, K. (2010). Tyrosine kinase 2 interacts with the proapoptotic protein Siva-1 and augments its apoptotic functions. Biochem Biophys Res Commun 400, 252-257.

Spinicelli, S., Nocentini, G., Ronchetti, S., Krausz, L.T., Bianchini, R., and Riccardi, C. (2002). GITR interacts with the pro-apoptotic protein Siva and induces apoptosis. Cell Death Differ 9, 1382-1384.

Thiery, J.P., Acloque, H., Huang, R.Y., and Nieto, M.A. (2009). Epithelial-mesenchymal transitions in development and disease. Cell 139, 871-890.

Thompson, C.B. (1995). Apoptosis in the pathogenesis and treatment of disease. Science 267, 1456-1462.

Vaux, D.L., and Korsmeyer, S.J. (1999). Cell death in development. Cell 96, 245-254.

Vogelstein, B., Lane, D., and Levine, A.J. (2000). Surfing the p53 network. Nature 408, 307-310.

Vousden, K.H., and Lu, X. (2002). Live or let die: the cell's response 
to p53. Nat Rev Cancer 2, 594-604.

Walmsley, S.R., Chilvers, E.R., Thompson, A.A., Vaughan, K., Marriott, H.M., Parker, L.C., Shaw, G., Parmar, S., Schneider, M., Sabroe, I., et al. (2011). Prolyl hydroxylase 3 (PHD3) is essential for hypoxic regulation of neutrophilic inflammation in humans and mice. J Clin Invest 121, 1053-1063.

Xiao, H., Palhan, V., Yang, Y., and Roeder, R.G. (2000). TIP30 has an intrinsic kinase activity required for up-regulation of a subset of apoptotic genes. EMBO J 19, 956-963.

Xue, L., Chu, F., Cheng, Y., Sun, X., Borthakur, A., Ramarao, M., Pandey, P., Wu, M., Schlossman, S.F., and Prasad, K.V. (2002).
Siva-1 binds to and inhibits BCL-X(L)-mediated protection against UV radiation-induced apoptosis. Proc Natl Acad Sci U S A 99, 6925-6930.

Yan, J., Menendez, D., Yang, X.P., Resnick, M.A., and Jetten, A.M. (2009). A regulatory loop composed of RAP80-HDM2-p53 provides RAP80-enhanced $p 53$ degradation by HDM2 in response to DNA damage. J Biol Chem 284, 19280-19289.

Yoon, Y., Ao, Z., Cheng, Y., Schlossman, S.F., and Prasad, K.V. (1999). Murine Siva-1 and Siva-2, alternate splice forms of the mouse Siva gene, both bind to CD27 but differentially transduce apoptosis. Oncogene 18, 7174-7179. 